\title{
Perfil epidemiológico da hanseníase na região Nordeste do Brasil no período de 2016-
} 2020

\author{
Epidemiological profille of leprosy in Northeastern Brazil in the period 2016 to 2020 \\ Perfil epidemiológico de la lepra en el Noreste de Brasil en el período 2016-2020
}

Recebido: 13/11/2021 | Revisado: 20/11/2021 | Aceito: 22/11/2021 | Publicado: 02/12/2021

\author{
Carlos Antonio de Lima Filho \\ ORCID: https://orcid.org/0000-0001-5517-0347 \\ Universidade Federal de Pernambuco, Brasil \\ E-mail: cttoni2000@gmail.com \\ Wanuska Munique Portugal \\ ORCID: https://orcid.org/0000-0002-1485-2007 \\ Universidade Federal de Pernambuco, Brasil \\ E-mail: ga.portugal@hotmail.com \\ Arthur de Morais e Silva \\ ORCID: https://orcid.org/0000-0003-1673-5282 \\ Faculdade Integrada Tiradentes, Brasil \\ E-mail: arthurmoraissilva@hotmail.com \\ Kydja Milene Souza Torres de Araújo \\ ORCID: https://orcid.org/0000-0001-5258-8780 \\ Universidade de Pernambuco, Brasil \\ E-mail: kydjamilleny@hotmail.com \\ Amanda Oliveira Bernardino Cavalcanti de Albuquerque \\ ORCID: https://orcid.org/0000-0002-1011-8964 \\ Universidade de Pernambuco, Brasil \\ E-mail: amandaobernardino@ hotmail.com \\ Matheus Vinicius Barbosa da Silva \\ ORCID: https://orcid.org/0000-0003-1295-6301 \\ Universidade Federal de Pernambuco, Brasil \\ E-mail: matheushue30@gmail.com \\ Daniela de Lira Silva \\ ORCID: https://orcid.org/0000-0001-8145-2508 \\ Universidade Federal de Pernambuco, Brasil \\ E-mail: daniela.lirasilva@ufpe.br \\ Carlos Henrique Tenorio Almeida do Nascimento \\ ORCID: https://orcid.org/0000-0001-6676-1660 \\ Centro Universitário Brasileiro, Brasil \\ E-mail: henrique_almeida89@hotmail.com \\ Rafael Caruso Modesto \\ ORCID: https://orcid.org/0000-0001-9960-9438 \\ Faculdade Integrada Tiradentes, Brasil \\ E-mail: rafaelcaruso@live.com \\ Ana Beatriz Siqueira Portela Gomes \\ ORCID: https://orcid.org/0000-0002-9537-7147 \\ Faculdade Integrada Tiradentes, Brasil \\ E-mail: ana.beatriz@soufits.com.br \\ Cristiane Macedo Vieira \\ ORCID: https://orcid.org/0000-0003-0384-0630 \\ Universidade Federal de Pernambuco, Brasil \\ E-mail: cristianemacedov@yahoo.com.br
}

\begin{abstract}
Resumo
Objetivo: Avaliar o perfil epidemiológico da hanseníase na região nordeste do Brasil no período de 2016-2020. Metodologia: Trata-se de um estudo retrospectivo, descritivo com abordagem quantitativa sobre o perfil epidemiológico dos casos de hanseníase na região nordeste do Brasil no período de 2016 a 2020, com dados coletados no Sistema de Notificação de Agravos de Notificação no período de outubro a novembro de 2021. Resultados: Foram diagnosticados 67.070 de casos de hanseníase na região nordeste no período de estudo, com predominância do sexo masculino (56,2\%), faixa etária dos 40-49 (18,4\%), a classificação operacional mais encontrada foi a multibacilar (73,9\%), com forma clínica dimorfa (42,9\%), a maioria foi de casos novos $(79,5 \%)$ com a maioria dos pacientes evoluídos para cura clínica $(59,5 \%)$, sendo que a maioria do grau de incapacidade após cura estando em branco $(48,5 \%)$ seguido de grau zero
\end{abstract}


(32,2\%). Conclusão: Os resultados evidenciam para ações em saúde voltadas para o diagnóstico precoce da doença e para a educação continuada dos profissionais de saúde.

Palavras-chave: Hanseníase; Epidemiologia; Mycobacterium Leprae; Atenção à saúde.

\begin{abstract}
Objective: To evaluate the epidemiological profile of leprosy in northeastern Brazil in the period 2016-2020. Methodology: This is a retrospective, descriptive study with a quantitative approach on the epidemiological profile of leprosy cases in northeastern Brazil in the period from 2016 to 2020, with data collected in the Notifiable Diseases Notification System from October to November 2021. Results: 67,070 cases of leprosy were diagnosed in the northeast region during the study period, with a predominance of males $(56.2 \%)$, age group 40-49 (18.4\%), the most operational classification. found was multibacillary $(73.9 \%)$, with borderline clinical form $(42.9 \%)$, most were new cases $(79.5 \%)$ with most patients evolved to clinical cure $(59.5 \%)$, with the majority of the degree of disability after cure being blank (48.5\%) followed by degree zero (32.2\%). Conclusion: The results show for health actions aimed at the early diagnosis of the disease and for the continuing education of health professionals.
\end{abstract}

Keywords: Leprosy; Epidemiology; Mycobacterium leprae; Delivery of health care.

\title{
Resumen
}

Objetivo: Evaluar el perfil epidemiológico de la lepra en el noreste de Brasil en el período 2016-2020. Metodología: Se trata de un estudio descriptivo retrospectivo con enfoque cuantitativo sobre el perfil epidemiológico de los casos de lepra en el noreste de Brasil en el período de 2016 a 2020, con datos recolectados en el Sistema de Notificación de Enfermedades Notificables de octubre a noviembre de 2021. Resultados: 67,070 casos de lepra fueron diagnosticados en la región noreste durante el período de estudio, con predominio del sexo masculino (56,2\%), grupo etario 40-49 $(18,4 \%)$, la clasificación más operativa, encontrada fue multibacilar $(73,9 \%)$, con forma clínica limítrofe $(42,9 \%)$, la mayoría fueron casos nuevos $(79,5 \%)$ y la mayoría de los pacientes evolucionaron hacia la curación clínica (59,5\%), y la mayoría del grado de discapacidad después de la cura quedó en blanco $(48,5 \%)$ seguido del grado cero $(32,2 \%)$. Conclusión: Los resultados muestran acciones de salud dirigidas al diagnóstico precoz de la enfermedad y a la formación continua de los profesionales de la salud.

Palabras clave: Lepra; Epidemiología; Mycobacterium leprae; Atención a la salud.

\section{Introdução}

A hanseníase, conhecida na antiguidade como lepra, é uma doença infecto-contagiosa causada pela bactéria mycobacterium leprae, a doença atinge principalmente as mucosas, pele e os nervos periféricos, ocasionando lesões neurais, fazendo com que as pessoas acometidas por ela adquiram incapacidades graves, levando ao longo da vida o que (Ministério da Saúde, 2020). Segundo Monteiro et. al. (2017) é uma doença de longo período de incubação, variando de 2 a 10 anos, com baixa morbidade e com um alto poder de transmissão.

A transmissão ocorre quando uma pessoa com a forma infectante da doença, a multibacilar, que não está em tratamento, elimina a bactéria pelas vias aéreas superiores (Ministério da Saúde, 2019). Segundo o guia de vigilância em saúde (2019) o diagnóstico é feito pela avaliação clínica, por meio do exame dermatoneurológico e historia/condição de vida do paciente, e também feito por meio de exame laboratoriais, com a baciloscopia da pele e o histopatológico.

De acordo com Freitas el. al. (2017) devido a falta de conhecimento, a hanseníase era confundida com outras doenças de pele e venéreas, presepunhando que para haver a transmissão teria que ter contato físico, principalmente o sexual , portan to associando a doença a algo pecaminoso. No Brasil, seu histórico foi marcado pelo isolamento compulsório sofridos pelos doentes, essa prática foi encerrada apenas na década de 1980 com a introdução do tratamento poliquimioterápico, com a criação deste medicamento, também houve a transição do nome lepra para hanseníase, para diminuir o estigma e preconceito da doença (Loures et. al., 2017). Para Gonçalves et. al. (2018) a hanseníase está nos grupos das chamadas doenças negligenciadas, esse fato significa que pelo fato dela abater mais indivíduos pobre em países em desenvolvimento, existe um baixo interesse das indústrias para a elaboração de fármacos, equipamentos e tecnologias sobre a doença.

Segundo a Organização Mundial da Saúde (WHO, 2020) em 2019 foram reportados 202.185 novos casos da doença no mundo, desses, 29.936 (14,8\%) ocorrem nas região das américas, sendo somente o Brasil responsável por 27.864 (93,1\%) dos novos casos da região, levando em consideração os casos mundial, o Brasil fica sem segundo lugar na ocorrência de novos casos, 
ficando atrás apenas da Índia.

Para Silva et. al. (2020) no cenário Brasileiro as regiões sul e sudeste tiveram bom resultados na redução dos casos da hanseníase, diferente das regiões norte, nordeste e centro-oeste onde os casos de hanseníase ainda se encontram em um patamar elevado. Desde 2016 a região nordeste vem sendo responsável por uma boa parte dos casos de hanseníase diagnosticados no Brasil, no ano de 2019 dos cinco primeiros estados com maior número de casos, três eram da região nordeste, com destaque para o estado de maranhão que ficou em segundo lugar com 3.972 novos casos, ficando atrás apenas do Mato Grosso que somou 5.233 casos.

Perante o exposto, é de suma importância a realização de estudos epidemiológicos sobre a hanseníase, para compreender os fatores que contribuem para a continuidade da doença, e também para que gestores e profissionais de saúde busquem ações para o controle, prevenção e tratamento da doença. O objetivo deste trabalho foi avaliar o perfil epidemiológico da hanseníase na região do nordeste no período de 2016 a 2020.

\section{Metodologia}

Trata-se de um estudo retrospectivo, descritivo, com abordagem quantitativa. Para Hochman et. al. (2005) um estudo retrospectivo é realizado a partir de dados do passado, já o descritivo estuda determinados aspectos de doenças para conhecer uma nova doença ou agravos a saúde. Foram utilizados dados secundários do Sistema de Informações de Agravos de Notificação (SINAN), mediante ao acesso do Tabulador Genérico de Domínio Público (TABNET), na seção "epidemiologia e morbidade", partindo para o tópico "hanseníase". A coleta de dados foi realizada entre os meses de outubro e novembro de 2021.

Para alcançar o objetivo final do trabalho foram avaliadas as variáveis número de casos no período estudado como : sexo, faixa etária, classe operacional, forma clínica, modo de entrada, tipo de saída e avaliação da capacidade após a cura. A pesquisa bibliográfica foi realizada por meio da plataforma Google Académico, Scientific Electronic Library Online (SCIELO) e na Literatura Latino-Americana e do Caribe em Ciências da Saúde (LILACS). Foi utilizado o programa Google Planilhas para a análise estatística descritiva e para auxiliar na elaboração das tabelas.

Por serem dados de domínio público disponibilizados pelo Ministério da Saúde, não houve a necessidade de submeter o artigo ao Comitê de Ética em Pesquisa.

\section{Resultados e Discussão}

Os dados epidemiológicos apontaram que foram diagnosticados 67.070 casos de hanseníase na região nordeste, na tabela 1 é possível observar a disposição dos casos no período estudado. O gráfico 1 mostra que houve uma regularidade de casos no período de 2016 a 2019, porém apresentou uma baixa no ano de 2020, quando a região registrou 8.190 novos casos. Entretanto, devido a pandemia causada pelo novo coronavírus, esses dados podem não representar a realidade, já que durante a pandemia houve uma redução nos casos diagnosticados de hanseníase (FIOCRUZ, 2021). 
Tabela 1. Distribuição dos casos de hanseníase diagnosticados na Região nordeste do Brasil no período de 2016-2020.

\begin{tabular}{llll}
\hline Ano & Casos & \% \\
2016 & 13.812 & 20,6 & 22,5 \\
2017 & 15.059 & 22,5 & 22,2 \\
2018 & 15.117 & 12,2 & \\
2019 & 14.892 & $\mathbf{1 0 0}$ \\
\hline
\end{tabular}

\section{Fonte: SINANWEB/DATASUS.}

A Tabela 2 mostra a relação entre os casos diagnosticados em homens $(56,2 \%)$ e mulheres $(43,8 \%)$, também foram encontrados 6 casos que no banco de dados estavam ignorados.

Tabela 2. Distribuição dos casos de hanseníase diagnosticados na Região nordeste do Brasil no período de 2016-2020, segundo o sexo

\begin{tabular}{ccc}
\hline Sexo & Casos & \% \\
Homens & 37.719 & 56,2 \\
Mulheres & 29.345 & 43,8 \\
Ignorado & 6 & 0,0 \\
Total & $\mathbf{6 7 . 0 7 0}$ & $\mathbf{1 0 0}$ \\
\hline
\end{tabular}

Fonte: SINANWEB/DATASUS.

Os estudos de Miranzi et. al (2010), de Heinrichsen et al. (2004) e os de Basso e Silva (2017) mostram que existe uma diferença significativa dos casos da hanseníase distribuídos entre os sexos, igualmente os dados apresentados na Tabela 2 . O fato dos homens procurarem menos os serviços de saúde, e de estar mais propensos à infecção pelo Mycobacterium Leprae em seu ambiente profissional, também são fatores para o maior número de casos nesse grupo (Goiabeira et al). Segundo Barbosa et. al. (2014) os fatores construtores da identidade masculina também são fatores importante que acarreta pontos importante sobre o estudo da epidemiologia da hanseníase.

A Tabela 3 mostra os casos diagnosticados de hanseníase segundo a faixa etária, e a 4 mostra a evolução dos casos em crianças/adolescentes menores de 15 anos no período estudado. 
Tabela 3. Distribuição dos casos de hanseníase diagnosticado na Região nordeste do Brasil no período de 2016-2020, segundo a faixa etária.

\begin{tabular}{ccc}
\hline Idade & Casos & \% \\
$\mathbf{1 - 4}$ & 194 & 0,4 \\
$\mathbf{5 - 9}$ & 1.383 & 2,1 \\
$\mathbf{1 0 - 1 4}$ & 2.619 & 3,9 \\
$\mathbf{1 5 - 1 9}$ & 3.131 & 4,7 \\
$\mathbf{2 0 - 2 9}$ & 7.015 & 10,5 \\
$\mathbf{3 0 - 3 9}$ & 11.021 & 16,4 \\
$\mathbf{4 0 - 4 9}$ & 12.342 & 18,4 \\
$\mathbf{5 0 - 5 9}$ & 12.239 & 18,2 \\
$\mathbf{6 0 - 6 9}$ & 9.885 & 14,7 \\
$\mathbf{7 0 - 7 9}$ & 5.264 & 7,8 \\
$\mathbf{8 0 <}$ & 1.977 & 2,9 \\
Total & $\mathbf{6 7 . 0 7 0}$ & $\mathbf{1 0 0}$ \\
\hline
\end{tabular}

Fonte: SINANWEB/DATASUS.

Tabela 4. Distribuição dos casos de hanseníase diagnosticados na Região nordeste do Brasil no período de 2016-2020, em menores de 15 anos.

\begin{tabular}{lcc}
\hline Ano & Casos & \% \\
2016 & 975 & 23,2 \\
2017 & 1.002 & 23,9 \\
2018 & 939 & 22,4 \\
2019 & 853 & 20,3 \\
2020 & 427 & 10,2 \\
Total & $\mathbf{4 . 1 9 6}$ & $\mathbf{1 0 0}$ \\
\hline
\end{tabular}

Fonte: SINANWEB/DATASUS.

A Tabela 3 aponta uma maior incidência de casos em grupo de pessoas dos 30 aos 59 anos, somando juntos $53 \%$ dos casos. A hanseníase apresenta um longo período de incubação, isso associado com a demora no diagnóstico e início do tratamento são fatores para o aumento dos casos com a progressão da idade, principalmente em pessoas economicamente ativa (Basso e Silva, 2017). A Tabela 4 mostra que apesar do aumento dos casos de hanseníase em pessoas menores de 15 anos do ano de 2016 para 2017, teve-se uma redução dos casos a partir do ano 2018, a grande redução dos casos no ano de 2020 não deve ser utilizada como parâmetro, devido aos vários impactos causados pela pandemia do novo coronavírus. Segundo Araújo et al., (2004) a ocorrência de hanseníase na faixa etária de menores de 15 tem um grande valor epidemiológico, pois indica uma exposição precoce ao agente etiológico, tornando importante ponto para avaliação da sua transmissibilidade. A Tabela 5 mostra a 
classificação operacional da hanseníase no período estudado e a 6 mostra a forma clínica dos casos.

Tabela 5. Classificação operacional dos casos de hanseníase na Região nordeste do Brasil no período de 2016-2020

\begin{tabular}{ccc}
\hline Classe operacional & Casos & \% \\
Paucibacilar & 17.445 & 26,0 \\
Multibacilar & 49.558 & 73,9 \\
Ignorado & 67 & 0,1 \\
Total & $\mathbf{6 7 . 0 7 0}$ & $\mathbf{1 0 0}$ \\
\hline
\end{tabular}

Fonte: SINANWEB/DATASUS.

Tabela 6. Forma clínica dos casos de hanseníase na Região nordeste do Brasil no período de 2016-2020.

\begin{tabular}{ccc}
\hline Forma clínica & Casos & $\%$ \\
Indeterminada & 8.714 & 13,0 \\
Tuberculóide & 9.266 & 13,8 \\
Dimorfa & 28.788 & 42,9 \\
Virchowiana & 11.282 & 16,8 \\
Não classificada & 5.608 & 8,4 \\
Ignorado/Branco & 3.412 & 5,1 \\
Total & $\mathbf{6 7 . 0 7 0}$ & $\mathbf{1 0 0}$ \\
\hline
\end{tabular}

Fonte: SINANWEB/DATASUS.

A classificação operacional foi criada pela OMS, devido a sua simplicidade, é amplamente utilizada em programas de combate à hanseníase pelo mundo, inclusive no Brasil, o seu principal uso está associado com a operacionalização do tratamento. Essa classificação se baseia na quantidade de manchas na pele, aquelas pessoas com uma a cinco manchas são classificadas como paucibacilar, já as que apresentam mais de seis é classificada como multibacilar (Word Health Organization, 1998). Como mostra na tabela 5 a classe operacional predominante na região no período estudado é a multibacilar, responsável por 73,9\% dos casos, para Gonçalves et. al. (2018) esse episódio está associado com a detecção tardia da doença, que contribui para manter a transmissibilidade da doença e também está relacionado a danos neurológicos causados pela doença.

Algumas pessoas acometidas pela hanseníase não apresentam lesões que possam ser totalmente visíveis, por isso, o ministério da saúde também usa outra ferramenta de classificação para auxiliar o diagnóstico, a classificação de madri, que avalia a forma clínica da doença, sendo a dimorfa aparecendo na maioria dos casos, formas clínicas paucibacilar (indeterminada e tuberculóide) são as que apresentam menor gravidade, já as multibacilar (dimorfa e virchowiana) são as mais graves e que pode causar incapacidades físicas (BRASIL, 2017)

Como mostrado na tabela 5 e 6, a maioria dos pacientes são classificados como multibacilares de forma clínica dimorfa, esses resultados vão em encontro com estudos de Vieira at. al. (2014), Rosa at. al. (2019) e de Novato et. al. (2020). Segundo Silva et. al. (2014), pacientes com essa condição tem um alto poder de transmissibilidade da doença e também se enquadram de maior gravidade da doença. A Tabela 7 mostra o modo de entrada dos pacientes. 
Tabela 7. Modo de entrada dos casos de hanseníase na região nordeste do Brasil no período de 2016-2020.

\begin{tabular}{ccc} 
Modo de entrada & Casos & \% \\
Caso novo & 53.335 & 79,5 \\
Transferência do mesmo município & 1.942 & 2,9 \\
$\begin{array}{c}\text { Transferência de outro município } \\
\text { (mesma UF) }\end{array}$ & 1.971 & 2,9 \\
Transferência de outros estados & & 1,3 \\
Transferência de outro país & 872 & 0,1 \\
Recidiva & 46 & 5,2 \\
Outros ingressos & 3.469 & 7,8 \\
Ignorado/Branco & 5.254 & 0,3 \\
\hline
\end{tabular}

\section{Fonte: SINANWEB/DATASUS.}

Em relação ao modo de entrada, a tabela 7 mostra a predominância de casos novos da doença. Segundo Silva et. al. (2020) um quadro novo de hanseníase se caracteriza por uma pessoa que apresenta os sinais e sintomas característico da doença e que nunca recebeu um tratamento específico para a doença, ainda segundo ao autor, uma taxa alta de casos novos em comprado com as outras variáveis, indica que a cadeia de transmissão está ativa na região. Diagnóstico tardio, subnotificação de casos e a não realização do esquema terapêutico adequado podem ser explicações para essa característica, já que pessoas pessoas com hanseníase que se enquadra nos itens anteriores está transmitindo o bacilo ou volta a transmitir, que são fatores para a continuação da taxa de transmissão da doença.

A Tabela 8 destaca o tipo de saída dos casos de hanseníase no período estudado.

Tabela 8. Saída dos casos de hanseníase na região nordeste do Brasil no período de 2016-2020.

$\begin{array}{ccc}\text { Tipo de saída } & \text { Casos } & \text { \% } \\ \text { Cura } & 39.916 & 59,5 \\ \text { Transferência para o mesmo município } & 1.908 & 2,8 \\ \text { Transferência para outro município } & 2.883 & 4,3 \\ \text { Transferência para outro estado } & 821 & 1,2 \\ \text { Transferência para outro país } & 36 & 0,1 \\ \text { Óbito } & 1.036 & 1,5 \\ \text { Abandono } & 3.501 & 5,2 \\ \text { Erro diagnóstico } & 815 & 1,2 \\ \text { Não preenchido } & 16.154 & 24,1 \\ \text { Total } & \mathbf{6 7 . 0 7 0} & \mathbf{1 0 0}\end{array}$


Os dados apontam que a maioria dos casos concluiu para a cura. A alta por cura é caracterizada quando o paciente realiza a poliquimioterapia com o tempo e dose adequada, segundo a forma operacional da doença (Brasil, 2017). De acordo com Junior (2011) e Pereira et. al. (2012) o tratamento adequado contra a doença é um fator primordial para a cura do paciente, na interromper a cadeia de transmissibilidade, tornando uma ferramenta importante para o controle da doença, além disso, tem papel crucial na prevenção de incapacidades provocadas pela doença.

Tabela 9. Avaliação da incapacidade dos pacientes com hanseníase na região nordeste do Brasil no período de 2016 -2020.

\begin{tabular}{ccc}
\hline Avaliação da Incapacidade cura & Casos & $\%$ \\
Grau 0 & 21.619 & 32,2 \\
Grau I & 5.121 & 7,6 \\
Grau II & 1.986 & 3,0 \\
Não avaliado & 5.790 & 8,6 \\
Em branco & 32.554 & 48,5 \\
Total & $\mathbf{6 7 . 0 7 0}$ & $\mathbf{1 0 0}$ \\
\hline
\end{tabular}

Fonte: SINANWEB/DATASUS.

Segundo Sobrinho et. al. (2007) grau 0 (zero) é quando a hanseníase não causou comprometimento nos olhos, nas mãos ou pés, o grau I (um) é quando existe a diminuição ou perda da sensibilidade e o grau II (dois) é quando existe deformidade e/ou incapacidades de natureza lagoftalmo, garras, mãos e pés caídos, entre outros. Se levarmos em consideração apenas a avaliação em relação ao grau, observamos que a maioria dos pacientes evoluíram sem nenhuma incapacidade, essa informação vai de encontro com estudos de Sarmento et. al. (2015). Contudo, os dados que estão em branco ou não foram avaliados superam o de pacientes com grau 0 , esse fato, somado que a maioria dos casos é de pacientes com a classificação operacional multibacilar e forma clínica dimorfa, sugere que o número de pacientes que tiveram alguma incapacidade após a cura da hanseníase pode ser muito maior. Para Alves et. al. (2017) avaliar o grau de incapacidade é uma ferramenta eficiente para auxiliar os estudos da situação epidemiológica da hanseníase, e também é um indicador do seu controle.

\section{Conclusão}

A partir dos dados analisados, na região nordeste no período de 2016 a 2020, destaca a ocorrência em indivíduos do sexo masculino, adultos, com faixa etária dos 20 aos 59 anos. O maior quantitativo de casos multibacilares com forma clínica dimorfa revela que há um diagnóstico tardio da doença, que pode contribuir para aumento dos casos de incapacidade, porém o alto número de dados em branco referente à incapacidade ocasionada pela doença impede de avaliar a real situação dessa problemática.

Os resultados alcançados evidenciam a necessidade de ações mais firmes voltadas para o diagnóstico precoce da doença, que podem ser tanto de atividades de educação em saúde voltadas para os sinais e sintomas da doença, como também de educação continuada para os profissionais de saúde, deixando os mesmos aptos para o diagnóstico e na assistência ao tratamento adequado. Por fim, é de suma importância que haja a continuação de estudo epidemiológica sobre a hanseníase no Brasil, principalmente nas regiões onde tem um alto índice de casos da doença, também é importante que futuros trabalhos sobre essa temática explore principalmente questões sobre classificação operacional, forma clínica e grau de incapacidade, para que possamos analisar o real impacto da hanseníase sobe a população. 


\section{Referências}

Brasil. Ministério da Saúde (2020). O que é hanseníase? Governo Federal. https://www.gov.br/saude/pt-br/assuntos/saude-de-a-a$\mathrm{z} / \mathrm{h} /$ hanseniase\#: :text=A\%20dura\%C3\%A7\%C3\%A30\%20do\%20tratamento\%20varia,medicamentos\%20s\%C3\%A3o\%20seguros $\% 20 \mathrm{e} \% 20 \mathrm{eficazes}$.

Monteiro, M. J. S., Santos, G. M., Barreto, M. T. S., Silva, R. V. S., Jesus, R. L. R., \& Silva, H. J. N., (2017). Perfil epidemiológico de casos de hanseníase em um estado do nordeste brasileiro. Rev. Aten. Saúde, 15(54), 21-28, https://doi.org/10.13037/ras.vol15n54.4766

Brasil. Ministério da Saúde (2019). Guia de vigilância em saúde, volume único. (3a ed.), https://bvsms.saude.gov.br/bvs/publicacoes/guia_vigilancia_saude_3ed.pdf

Freitas. D. V., Xavier, S. S., \& Lima, M. A. T., (2017). Perfil Epidemiológico da Hanseníase no Município de Ilhéus-BA, no Período de 2010 a 2014. J Health Sci;19(4):274-7. https://pesquisa.bvsalud.org/portal/resource/pt/biblio-877799

Loures, L. F., Mármora, C. H. C., Barreto, J., \& Duppre, N. C., (2017). Percepção do estigma e repercussões sociais em indivíduos com hanseníase. Psicologia Em Estudo, 21(4), 665-675. doi.org/10.4025/psicolestud.v21i4.30037

Gonçalves, M., Prado, M. A. R., Silva, S. S., Santos, K. S., Araújo, P. N., \& Fortuna, C. M., (2018). Trabalho e hanseníase: as mulheres em suas dores, lutas e labutas. Rev. Bras. Enfermagem (internet). 2018;71(suppl 1):660-7. doi.org/10.1590/0034-7167-2017-0598

World Health Organization (WHO, 2020). Global leprosy (hansen disease) update 2019, time: too step-up prevention initiatives. Weekly Epidemiological Record, (95), 417-440, https://apps.who.int/iris/bitstream/handle/10665/334140/WER9536-eng-fre.pdf? sequence=1\&sequence=1\&isAllowed=y\&ua=1

Hochman, B., Nahas, F. X., Filho, R. S. O., \& Ferreira, L. M., (2005). Desenhos de pesquisas. Acta cirúrgica brasileira - 20 (2). https://www.scielo.br/j/acb/a/bHwp75Q7GYmj5CRdqsXtqbj/?format=pdf\&lang=pt

Fiocruz (2021). Durante a pandemia, diagnósticos de hanseníase caem $40 \%$ no Rio. https://portal.fiocruz.br/noticia/durante-pandemia-diagnosticos-dehanseniase-caem-40-no-rio

Miranzi, S. S. C., Pereira, L. H. M., \& Nunes, A. A. (2010). Perfil epidemiológico da hanseníase em um município brasileiro, no período de 2000 a 2006. Revista da Sociedade Brasileira de Medicina Tropical 43(1): 62-67. https://doi.org/10.1590/S0037-86822010000100014

Goiabeira, Y. N. A., Rolim, I. L. T. P., Aquino, D. M. C., Inácio, A. S., \& Queiroz, R. C. S. (2018). Perfil Epidemiológico e clínico da hanseníase em capital hiperendêmica. Rev. enferm. on line., Recife, 12(06): 1507-13. https://doi.org/10.5205/1981-8963-v12i6a234693p1507-1513-2018

Barbosa, D. R. M., Almeida, M. G., \& Santos. A. G., (2014). Caracteristocas epidemiologicas e espaciais da hanseníase no Estado do Maranhão, Brasil, 20012012. Medicina (Ribeirão Preto) 47 (4): 347-56. dói: https://doi.org/10.11606/issn.2176-7262.v47i4p347-356

Hinrichsen, S. L., Rolim, H., Pinheiro, M. R. S., \& Danda, G. J. N. (2004). Aspectos epidemiológicos da hanseníase na cidade de Recife, PE em 2002. An bras Dermatol, 79(4): 413-421, https://doi.org/10.1590/S0365-05962004000400003

Basso, M. E. M., \& Silva, R, L, F. Perfil clínico-epidemiológico de pacientes acometidos pela hanseníase atendidos em uma unidade de referência (2017). Rev. Soc. Bras. Clin. Med. 15(1): 27-32. https://www.sbcm.org.br/ojs3/index.php/rsbcm/article/view/247

Araújo, M.G., Lana, F. C. F., Fonseca, P. T. F., \& Lanza, F. M. (2004). Detecção da hanseníase na faixa etária de 0 a 14 anos em Belo Horizonte no período 1992-1999: implicações para o controle. Revista médica de Minas Gerais, 14(2): 78-83. http://rmmg.org/artigo/detalhes/1498\#: :text=Em\%20Belo\%20Horizonte\%2C\%2039\%25\%20dos,\%2C14\%2F10.000 habitantes).

World Health Organization (WHO, 1998). Who expert committee on leprosy. Who Technical Report Series, Genebra, p. 52, 1998: https://www.google.com/url?sa=t\&source=web\&rct=j\&url=https://www.who.int/lep/resources/Expert.pdf\&ved=2ahUKEwibu5X13430AhX6GLkGHZMdCD 0QFnoECBQQAQ\&usg=AOvVaw13diIJ-i1jK1RKiNnX1U8z

Silva, M. S., Silva, E. P., Monteiro, F. F., \& Teles, S. F. (2014). Perfil clínico-epidemiológico da hanseníase no estado do Acre: estudo retrospectivo. Hansenologia Internationalis: Hanseníase $\quad$ E $\quad$ Outras https://periodicos.saude.sp.gov.br/index.php/hansenologia/article/view/36180

Rosa, A. M.; Souza, B. A. B., Jesus, G, M, P., Torres, I. L., Souza, G. C., Rodrigues, H. R., \& Vieira, F. S. (2019). Perfil clínico-epidemiológico da hanseníase no interior maranhense no período de 2013 a 2017. Prevenção e promoção de saúde, 20143 ed. Ponta Grossa: Atena Editora, 2019 ; 28 p. https://www.atenaeditora.com.br/post-artigo/26109

Gonçalves, N. V., Alcântara, R. C. C., Junior, A. S. S., Pereira, A. L. R. R., Miranda, C. S. C., Oliveira, J. S. S., Melo, A. C. B. V. Guedes, J. A., Costa, R. J. F., Costa, S. B. N., Marcos, W., Gomes, R. P., Oliveira, R. A. C., \& Palácios, V. R. C. M.,. (2018). A hanseníase em um distrito administrativo de Belém, estado do Pará, Brasil: relações entre território, socioeconomia e política pública em saúde, 2007-2013. Revista Pan-Amazônica de Saúde, 9(2), 21-30. https://dx.doi.org/10.5123/s2176-62232018000200003

Brasil, Ministério da Saúde, Secretaria de Vigilância em Saúde, Departamento de vigilância das doenças transmissíveis (2017). Guia Prático sobre a hanseníase, Ed. 1 (versão eletrônica). http://bvsms.saude.gov.br/bvs/publicacoes/guia_pratico_hanseniase.\%0D\%0Apdf

Vieira, G. D., Aragoso, I., Carvalho, R. M. B., \& Souza, C. M., (2014). Hanseníase em Rondônia: incidência e características dos casos notificados, 2001 a 2012. Epidemiol. Serv. Saúde, 23(2), 269-275, http://dx.doi.org/10.5123/S1679-49742014000200008

Novato, K. M., Grangeiro, A. M., Mello. B. C., \& Fagundes, F. R. Q. O., (2020). Perfil epidemiológico da hanseníase no estado do Tocantins no período de 2014 a 2016. Revista de Patologia do Tocantins. 6(4): 27-31. https://sistemas.uft.edu.br/periodicos/index.php/patologia/article/view/8008 
Silva, L. S. R., Miritiba, C. S., Silva, K. J. S., Pessoa, I. M., Silva, A. S., Jardim, M. J. A., Cardoso, L. S. P., Cunha, N. G. T., Oliveira, L. S., \& Rodrigues, R. L., (2020). Perfil clinico e epidemiológico dos casos de hanseníase em pacientes adultos em um municipio do maranhão. REAS/EJCH 12(9): e3902. https://doi.org/10.25248/reas.e3902.2020

Pereira, D. L., Brito, L. M., Nascimento, A. H., Ribeiro, E. L., Lemos, K. R. M., Alves, J. N., \& Brandão, L. C. G., (2012). Estudo da prevalência das formas clínicas da hanseníase na cidade de Anápolis-GO. Ensaios e Ciência: Ciências Biológicas, Agrárias e da Saúde, 16(1), 55-67. https://www.redalyc.org/articulo.oa?id=26025372004

Junior, F. A. C. P., (2011). Motivos do abandono ou interrupção do tratamento da hanseníase: uma revisão sistemática da literatura. 24f. TCC (Especialização em Sistema e serviços de saúde) - Centro de Pesquisa Ageu Magalhães, recife 2011. https://www.arca.fiocruz.br/handle/icict/28224

Sobrinho, R. A. S., Mathias, T. A. F., Gomes, E. A., \& Lincoln, P. B., (2007). Avaliação do grau de incapacidade em hanseníase: uma estratégia para sensibilização e capacitação da equipe de enfermagem. Rev. Latino-Am. Enfermagem 15(6). https://doi.org/10.1590/S0104-11692007000600011

Sarmento, A. P. A., Pereirão, A. M., Ribeiro, F., Castro, J. L., Almeida, M. B., \& Ramos, N. M., (2015). Perfil epidemiológico da hanseníase no priodo de 2009 a 2013 no município de Montes Claro (MG). Rev. Soc. Bras. Clin. Med. 13(3);180-4. http://files.bvs.br/upload/S/1679-1010/2015/v13n3/a5389.pdf

Alves, E.S., Oliveira, L. B., Araújo, T. M. E., Melo, I. V., Araújo, R. P. S., \& Marques, L. M. F., (2017). Perfil epidemiológico da hanseníase em um município do nordeste brasileiro: uma analise retrospectiva. Rev. Fund. Care Online. 9(3):648-652. https://www.redalyc.org/pdf/5057/505754116007.pdf 